\title{
IGF-1 Conjugated Sub-5 nm Ultrafine Iron Oxide Nanoparticle Enhances Targeted Drug Delivery and Efficacy of DNA Topoisomerase Inhibitor SN38 for Pancreatic Cancer: An in Vitro Study
}

\section{Peijia Xu}

Affiliated Longhua People's Hospital, Southern Medical University

\section{Ting Xue}

Affiliated Longhua People's Hospital, Southern Medical University

Jonathan Padelford

$5 \mathrm{M}$ Biomed, LLC

\section{Xingkui Xue}

Affiliated Longhua People's Hospital, Southern Medical University

Alyssa Y Wu

Emory University

Yuancheng Li

$5 \mathrm{M}$ Biomed, LLC

Liya Wang ( $\sim 2718377613 @ q q . c o m)$

Affiliated Longhua People's Hospital, Southern Medical University

\section{Research Article}

Keywords: Ultrafine iron oxide nanoparticle, Drug delivery, Pancreatic cancer, SN38, Targeting

Posted Date: January 3rd, 2022

DOI: https://doi.org/10.21203/rs.3.rs-1150109/v1

License: (c) (i) This work is licensed under a Creative Commons Attribution 4.0 International License.

Read Full License 


\section{Abstract \\ Background}

Pancreatic cancer remains one of the most lethal cancers largely due to the inefficient delivery of therapeutics. Nanomaterials have been extensively investigated as drug delivery platforms, showing improved drug pharmacodynamics and pharmacokinetics. However, their applications in pancreatic cancer have not yet been successful due to limited tumor delivery caused by dense tumor stroma and distorted tumor vasculatures. Meanwhile, smaller-sized nanomaterials have shown improved tumor delivery and retention in various tumors, including pancreatic tumors, suggesting their potential in enhancing drug delivery.

\section{Methods}

An ultrafine iron oxide nanoparticle (uIONP) was used to encapsulate 7-ethyl-10-hydroxyl camptothecin (SN38), the water-insoluble active metabolite of chemotherapy drug irinotecan for treating pancreatic cancer in clinic. Insulin-like growth factor 1 (IGF-1) was conjugated to ulONP as a ligand for targeting pancreatic cancer and stromal cells overexpressing IGF-1 receptor (IGF1R). The SN38 loading and release profile were characterized. The cancer cell targeting and induced apoptosis by developed nanoformulationIGF1-uIONP/SN38 were also investigated.

\section{Results}

IGF1-uIONP/SN38 demonstrated stable drug loading in physiological pH with the loading efficiency of $68.2 \pm 3.5 \%$ (SN38/Fe, wt\%) and $<7 \%$ release for 24 hours. In tumor-interstitial- and lysosomal-mimicking $\mathrm{pH}$ (6.5 and 5.5), 52.2 and $91.3 \%$ of encapsulated SN38 were released over 24 hours. The IGF1ulONP/SN38 exhibited specific receptor-mediated cell targeting and cytotoxicity to MiaPaCa-2 cells with IC50 of $11.8 \pm 2.3 \mathrm{nM}$, but not to HEK293 human embryonic kidney cells.

\section{Conclusion}

The IGF1-ulONP significantly improved the delivery of SN38 to targeted pancreatic cancer cells, holding the potential for in vivo theranostic applications.

\section{Introduction}

Pancreatic ductal adenocarcinoma (PDAC), generally referred as pancreatic cancer, represents the majority of malignant neoplasms of the pancreas with a 5 -year survival rate $<7 \%[1]$. As only $10-15 \%$ of patients are suitable for surgical resection, chemotherapy plays a critical role in the clinical management of pancreatic cancer[2]. However, gemcitabine, used as a first-line chemotherapy for pancreatic cancer for 
decades[3], has not achieved satisfactory clinical outcomes, due to its poor stability in plasma, inefficient uptake by cancerous cells and deactivation by blood and hepatic cells[4]. Another first-line chemotherapy regimen consisting of 5-fluorouracil, folinic acid, irinotecan and oxaliplatin, namely FOLFIRINOX, achieved better patient survival than gemcitabine, but adverse side effects were reported[4]. Nanomaterial-based drug delivery systems, at the meantime, demonstrated advantages in improving the delivery efficiency of drugs through enhanced permeability and retention (EPR) driven passive targeting and ligand-mediated active targeting to tumors, and preventing the drugs from premature release, thus reducing the systemic toxicity $[5,6]$. Several US Food and Drug Administration (FDA) approved nano-formulations for pancreatic cancer, such as albumin-bound paclitaxel (nab-paclitaxel)[7] and liposomal irinotecan formulation MM398[8], have shown increased tumor delivery of drugs and improved inhibition of tumor growth in preclinical studies[9], and more beneficial for patient survival in clinical trials[7, 10]. However, the effectiveness of nanomaterial-based formulation was impeded, largely due to the pathological barriers of pancreatic cancer. For example, the desmoplastic stroma with resulted solid stress and interstitial fluid pressure prevent the nano-formulations from reaching the cancer cells in the tumor parenchyma[11, 12]. The hypovascularity of the pancreatic tumor causes limited fenestrations of tumor blood vessels, lowering the delivery of nano-formulations to the tumor[13].

The size of nanomaterials has been shown critical for efficient tumor penetration to improve the treatment efficacy. On a mouse model of pancreatic tumor featuring extensive stroma and poor vascularization with reduced permeability, a $30 \mathrm{~nm}$ micelle demonstrated significantly improved tumor accumulation and anti-tumor activity comparing to the larger counterparts (up to $100 \mathrm{~nm}$ )[14]. Iron oxide nanoparticles (IONPs) with sizes typically ranging from 10 to $50 \mathrm{~nm}$ have been extensively investigated as drug delivery systems for a variety of cancers $[15,16]$, including pancreatic cancer[17, 18], owing to its biocompatibility, drug loading capability and capacity as imaging probes[19-23]. With the size further reduced to the sub- $5 \mathrm{~nm}$ range, ultrafine IONP (uIONP) with a diameter of $3.5 \mathrm{~nm}$ demonstrated severalfold enhancement in passive targeting[16] and ligand-mediated active targeting[24] for solid tumors, comparing to the IONPs with core sizes of 20 and $30 \mathrm{~nm}$. Hence, uIONP holds great potential to penetrate pancreatic tumors more easily than current larger-sized nano-formulations. In addition, the greater specific surface area of uIONPs allows for a substantially higher drug loading efficiency [25].

Here, we report the application of uIONPs with an amphiphilic polymer coating as carriers to deliver DNA topoisomerase I inhibitor 7-ethyl-10-hydroxyl camptothecin (SN38), the active metabolite of irinotecan with poor water solubility but much greater potency than irinotecan[26, 27]. Insulin-like growth factor 1 (IGF-1) was conjugated to the uIONP with SN38 loading (IGF1-ulONP/SN38) as the ligand to target the IGF-1 receptor (IGF1R), which is highly upregulated on the surface of pancreatic cancer cells and stromal components[28, 29] but not in normal pancreas[17]. The IGF1-uIONP/SN38 was characterized for the SN38 loading efficiency and release profile under different conditions. The pancreatic cancer cell targeting by IGF1-ulONP/SN38 and the induced cell apoptosis were also investigated.

\section{Methods}




\section{Encapsulation of SN38 to ulONPs}

SN38 $(10 \mathrm{mg})$ was dissolved in DMSO $(0.5 \mathrm{~mL})$ before mixing with the ulONP solution in water $(1 \mathrm{mg}$ $\mathrm{Fe} / \mathrm{mL}$ ) in the weight ratio of 1:1 (Fe/SN38). The mixture was incubated for 24 hours at room temperature. PEG550 (1 mL) was then added. The mixture was further incubated for 24 hours at room temperature, and then centrifuged at $3000 \mathrm{rpm}$ for $5 \mathrm{~min}$ to precipitate the free SN38. The supernatant was collected and centrifuged using an Amicon ultra-4 centrifugal filter unit to remove the free PEG550. The residual uIONP/SN38 was re-suspended in water, and the filtration/re-suspension cycle was repeated three times.

\section{Loading Efficiency and Release Profile of IGF1-UIONP/SN38}

The SN38 loading efficiency on uIONPs was defined as the weight\% of SN38 on ulONPs by equation (1):

$$
\text { Loading Efficiency }=\frac{\text { Mass of } S N 38}{\text { Mass of utONP }} \times 100 \% \quad \text { equation (1) }
$$

where the Mass of SN38 and Mass of uIONP were the amounts of SN38 and Fe in mg, respectively, in a given volume of ulONP/SN38 solution. The absorbance of SN38 at $380 \mathrm{~nm}$ was measured on a Genesys 50 UV-Vis spectrophotometer (Thermo Scientific, Waltham, MA, USA) for quantify SN38 following a

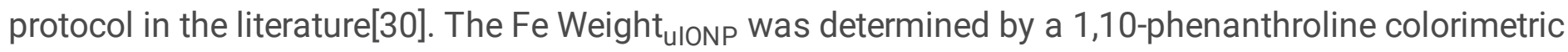
assay following the method described previously[31].

The SN38 release profile was determined by incubating uIONP/SN38 in buffered solutions of pH 5.5, 6.5 and 7.4, mimicking the lysosomal[32], tumor interstitial[33] and physiological environment, respectively. Briefly, uIONP/SN38 was incubated in acetate buffer $(0.1 \mathrm{M}, \mathrm{pH} 5.5)$, potassium phosphate buffer $(1 \mathrm{M}, \mathrm{pH}$ 6.5) and PBS (1X, pH 7.4) with the Fe concentration of $1 \mathrm{mg} / \mathrm{mL}$ for $1,2,4,8$ and 24 hours. The samples were then centrifugation at $3000 \mathrm{rpm}$ for $10 \mathrm{~min}$ to allow the released SN38 to precipitate. The supernatant was then collected to measure the loading efficiency of SN38 using the method described above. Each measurement was repeated three times.

\section{Conjugation of IGF-1 and NIR830 Dye to ulONPs}

uIONP/SN38 was firstly conjugated with IGF-1 through sulfo-SMCC following the established procedure[34]. uIONP/SN38 (2 mg Fe/mL, $0.5 \mathrm{~mL})$ was mixed with PBS $(0.5 \mathrm{~mL})$. Sulfo-SMCC $(1 \mathrm{mg})$ was then added to incubate with uIONP/SN38 for two hours at room temperature to allow the reaction with amine groups on the surface of uIONP. Afterwards, un-reacted sulfo-SMCC was removed by a PD-10 desalting column. To the collected SMCC-uIONP/SN38, IGF-1 was added in the molar ratio of 20:1 (IGF1/uIONP). The solution was incubated at room temperature for one hour to allow the coupling of IGF-1. The NIR830 dye was made as a NHS ester (NHS-NIR830), which readily reacted with amine groups on the surface of uIONP, following the method in the literature with IR-783 as the starting material[35]. For the coupling reaction, NHS-NIR830 was added to the IGF1-uIONP/SN38 in the molar ratio of 20:1 
(NIR830/ulONP). The mixture was incubated for two hours at room temperature, before removing free NHS-NIR830 by a PD-10 desalting column to yield NIR830-IGF1-uIONP/SN38. The hydrodynamic diameters and zeta-potentials of uIONP/SN38 before and after the functionalizations were measured on a Malvern Zetasizer NanoZS90 (Malvern, United Kingdom).

\section{In Vitro Cancer Cell Targeting of IGF1-ulONP/SN38}

In an 8-well chamber slide, $5 \times 10^{4}$ MiaPaCa-2 and HEK293 cells (Procell Life Science\&Technology Co., Ltd., Wuhan, China) were seeded and cultured for 24 hours in DMEM and EMEM, respectively, supplemented with $1 \%$ penicillin-streptomycin and $10 \%$ FBS. The cells were then incubated with NIR830IGF1-uIONP/SN38 and NIR830-uIONP/SN38 for three hours at $37^{\circ} \mathrm{C}$ with the Fe concentration of 0.02 $\mathrm{mg} / \mathrm{mL}$. Afterwards, the cells were washed with PBS for three times, and fixed with $4 \%$ paraformaldehyde. After washing with PBS three times again, the cells were mounted with ProLong ${ }^{\text {TM }}$ Gold Antifade Mountant with DAPI, before fluorescence imaging on a fluorescent microscope (BZ-X710, Keyence, Osaka, Japan) with a customized filter for NIR830.

\section{Cytotoxicity Assays of IGF1-UIONP/SN38}

The cytotoxicity assays of IGF1-uIONP/SN38, uIONP/SN38 and free SN38 for MiaPaCa-2 and HEK293 cells was performed using an AlamarBlue assay kit following the manufacturer's protocol. In a 96-well plate, 2000 cells/well were seeded and cultured for 24 hours to allow the attachment of cells. IGF1ulONP/SN38, uIONP/SN38 and free SN38 (20 mM dissolved in DMSO) were added to each well to incubate with the cells at $37^{\circ} \mathrm{C}$ for 72 hours with the SN38 concentrations of $0,2.5,4.9,9.8,19.5,39.1$, $78.1,156.2,312.5,625,1250$ and $2500 \mathrm{nM}$. After three washes with cold PBS, the cells were measured for viability using AlamarBlue assay. Results were normalized according to the cells treated with no SN38. Data was processed using Origin software (Originlab Corporation, Northampton, MA, USA).

\section{Induction of Cell Apoptosis by IGF1-ulONP/SN38}

On 6-well plates, $10^{5} \mathrm{MiaPaCa}-2$ cells were seeded in each well and cultured in $1 \mathrm{~mL}$ DMEM supplemented with $1 \%$ penicillin-streptomycin and $10 \% \mathrm{FBS}$ for 24 hours at $37^{\circ} \mathrm{C}$ to allow the cells to attach. Afterwards, the culture media were replaced with fresh media containing IGF1-uIONP/SN38, ulONP/SN38 and free SN38 at the SN38 dosages of 1,5 and $10 \mu \mathrm{M}$. Cells treated with medium only were used as the control. The cells were incubated for additional 24 hours, before removing from the wells by trypsin-EDTA. The removed cells were collected and washed three times with PBS, and stained for annexin $\mathrm{V}$ and 7-AAD using the Annexin $\mathrm{V}$ Apoptosis kit. The cells were analyzed using a BD FACSymphony A3 flow cytometer. Measurements were repeated three times for $1 \times 10^{5}$ cells each time. The data was processed by Tree Star FlowJo software (FlowJo, LLC, Ashland, OR, USA).

\section{Statistical Analysis}

Data was presented in the format of mean \pm standard deviation. Statistical significance was determined by a two-tailed unpaired $t$ test when comparing the hydrodynamic diameters and zeta-potentials of 
uIONPs before and after SN38 loading and conjugation of IGF-1. The level of significance was set at $p<$ 0.05 .

\section{Results}

\section{Characterization of SN38 Loaded ulONPs}

The commercial amphiphilic polymer coated ulONPs with an averaged diameter of $3.5 \mathrm{~nm}$ was used for encapsulating SN38. The ulONP demonstrated a narrow distribution of hydrodynamic diameter measured by dynamic light scattering (Fig. 1a), indicating monodispersed ulONPs with a highly uniformed size, which was further supported by the transmission electron microscopic (TEM) image (Fig. 1a, inset). After mixing SN38 with uIONPs to allow the accumulation of SN38 in the hydrophobic moiety of the coating polymer, PEG550 was added to further stabilize the loading of SN38. The emerging peak at $\sim 380 \mathrm{~nm}$ for ulONP/SN38 in the UV-Vis spectrum, comparing to that of uIONP alone and free SN38 peaked at $\sim 405 \mathrm{~nm}$ (Fig. 1b), indicated the loading of SN38 to the amphiphilic polymer coated ulONPs[30]. Worth noting, the peak of SN38 blue shifted after encapsulation, possibly due to the ionic hydrogen bonding between the Fe and SN38 molecules[36].

The loading efficiency of SN38 was measured to be $68.2 \pm 3.5 \%$ and found stable in PBS (pH 7.4), with a $<7 \%$ variation over 24 hours (Fig. 1c), suggesting the capability of ulONP/SN38 in preventing the premature drug release during blood circulation. At $\mathrm{pH}$ of 6.5 and 5.5 , which mimicked the acidic conditions in tumor interstitium[33] and lysosome[32], 47.8\% (pH 6.5) and 9.7\% (pH 5.5) of SN38 remained encapsulated over 24 hours (Fig. 1c), indicating the potential environment-triggered drug release in the tumor interstitial space and the lysosomes of cancer cells after IGF1-uIONP/SN38 was internalized through receptor-mediated endocytosis[37]. The release profiles of uIONP/SN38 showed a plateau-like stages from one to two hours after exposing to the acidic solutions, indicating a stepwise SN38 release. As the addition of PEG550 further increased the SN38 loading efficiency comparing to that without PEG550 (48.3 $\pm 6.1 \%$ ), which showed no plateau-like stage in the release profile (Supporting Information, Fig. s1), the release pattern in Fig. 1c can be ascribed to the fast release of PEG550 with the loosely encapsulated SN38 molecules within one hour, and a subsequent release of SN38 from the inner layer of coating polymer.

\section{Optimization and Functionalization of uIONP/SN38}

To investigate the effect of PEG molecules in stabilizing the SN38 loading, PEG with different molecular weights were tested in SN38 encapsulation. The loading efficiencies of SN38 decreased from $68.2 \pm 3.5 \%$ to $42.1 \pm 3.8 \%$, as the molecular weights of PEG increased from 550 to $5000 \mathrm{~g} / \mathrm{mol}$ (Fig. 2a), indicating a better SN38 loading by the PEG with a lower molecular weight. With PEG550 selected for optimal loading of SN38, the encapsulation of SN38 did not result in a significant $(p>0.05)$ change on the hydrodynamic diameter (Fig. 2b) or the zeta-potential (Fig. 2c). The uIONP/SN38 was then functionalized with IGF-1 as the targeting ligand for IGF1R, whose expression was highly upregulated in both pancreatic cancer cells and stromal component. The significantly $(p<0.001)$ increased hydrodynamic diameters of ulONP/SN38 
$(9.9 \pm 1.8 \mathrm{~nm})$ to IGF-uIONP/SN38 $(14.5 \pm 2.1 \mathrm{~nm})$ indicated the conjugation of IGF-1 (Fig. 2b), which was further confirmed by the change of zeta-potentials from the positive surface charge for ulONP/SN38 $(0.99 \pm 0.31 \mathrm{mV})$ to the negative charge $(-0.88 \pm 0.46 \mathrm{mV})$ after conjugation of IGF-1 (Fig. $2 \mathrm{c}$ ).

\section{Targeting of Pancreatic Cancer Cells by IGF1-ulONP/SN38}

To validate the targeting effect of IGF1-uIONP/SN38 to pancreatic cancer cells, near infrared (NIR) dye NIR830 labeled IGF1-uIONP/SN38 was incubated with MiaPaCa-2 human pancreatic cancer cells with overexpression of IGF1R[28, 29], with HEK293 human embryonic kidney cells with normal expression of IGF1R[17] as the control cell line. NIR830-IGF1-uIONP/SN38 at the Fe concentration of $0.02 \mathrm{mg} / \mathrm{mL}$ was applied to demonstrate the difference in cellular uptake of NIR830-IGF1-ulONP/SN38 by MiaPaCa-2 and HEK293 cells. Extensive NIR signal was observed on MiaPaCa-2 cells (Fig. 3a to c), indicating a substantial uptake of NIR830-IGF1-ulONP/SN38 by the cancer cells. In contrast, NIR830-IGF1uIONP/SN38 showed undetectable NIR signal on HEK293 cells (Fig. 3d to f). The IGF1-mediated pancreatic cancer cell targeting was further validated by incubating MiaPaCa-2 cells with non-targeted ulONP/SN38, in which no MiaPaCa-2 cell targeting was observed for NIR830-uIONP/SN38 when IGF-1 was not conjugated as the targeting ligand (Fig. $\mathbf{3 g}$ to i).

\section{Cytotoxicity of IGF1-ulONP/SN38}

The cytotoxicity of IGF1-uIONP/SN38 for targeted pancreatic cancer cells was investigated by measuring the viability of MiaPaCa-2 cells after incubating with IGF1-uIONP/SN38 for 72 hours at $37^{\circ} \mathrm{C}$ with the SN38 concentration ranging from 0 to $2500 \mathrm{nM}$. IGF1-ulONP/SN38 demonstrated a greater inhibitory effect on the growth of MiaPaCa-2 cell than those treated with free SN38 and non-targeted treatment uIONP/SN38, which showed little toxicity (Fig. 4a). The absolute half-maximal inhibitory concentration $\left(\mathrm{IC}_{50}\right)$ of IGF1-uIONP/SN38 for MiaPaCa-2 cells was calculated to be $11.8 \pm 2.3 \mathrm{nM}$ (Fig. 4a). In comparison, MiaPaCa-2 treated with free SN38 reached a viability of $\sim 50 \%$ at $78.1 \mathrm{nM}$ with no further inhibition on cell growth at higher SN38 concentration (Fig. 4a), suggesting the limit of cytotoxicity induced by free SN38 under the experimental condition. As 20\% cells remained viable after treating with IGF1-uIONP/SN38 at the SN38 concentration of 39.1 to $2500 \mathrm{nM}$, it can be concluded that IGF1-ulONP as a drug carrier improved the delivery efficiency of SN38 to the pancreatic cancer cells than the free drug, and subsequently enhanced the efficacy of SN38 in inhibiting the cell growth. Meanwhile, IGF1ulONP/SN38 did not exhibit obvious cytotoxicity for HEK293 cells at most SN38 concentrations, with $\sim 10 \%$ reduction of cell viability at the concentration of $2500 \mathrm{nM}$ (Fig. 4 b), suggesting the biosafety of IGF1-uIONP/SN38 for non-targeted cells in healthy organs.

\section{Enhancement of Pancreatic Cancer Cell Apoptosis by IGF1-ulONP/SN38}

To quantitatively assess the SN38 induced cell apoptosis after the enhanced cancer cell delivery by IGF1uIONP, IGF1-uIONP/SN38 treated MiaPaCa-2 cells were analyzed by fluorescence assisted cell sorting (FACS) after the fluorescence labeling using an Annexin V Apoptosis kit. After incubating with IGF1ulONP/SN38 for 24 hours with the SN38 concentrations of 1, 5 and $10 \mu \mathrm{M}$, the MiaPaCa-2 cells did not 
show a dose-dependent change on the populations of dead $\left(A n n e x i n V^{+} / 7-A A D^{+}\right)$, apoptotic $\left(A n n e x i n V^{+} / 7-\right.$ $\left.A A D^{-}\right)$and live (AnnexinV $\left.V^{-} / 7-A A D^{-}\right)$cells, with the population of dead cells varying from $80.2 \pm 0.9 \%$ to $82.2 \pm 1.5 \%$ (Fig. 5 a to d). Meanwhile, MiaPaCa-2 cells treated with free SN38 under the same condition exhibited increased populations of dead cells from $41.8 \pm 1.2 \%$ to $53.0 \pm 1.4 \%$ and decreased live cell population from $31.7 \pm 1.0 \%$ to $23.9 \pm 1.2 \%$, when SN38 concentration was increased from 1 to $10 \mu \mathrm{M}$

(Fig. 5a, e to g). These results suggested that the dosage for IGF1-ulONP/SN38 to be effective in inducing cell apoptosis was remarkably lower than that for free SN38, and IGF1-uIONP/SN38 possessed an approximate 1-fold better efficacy than free SN38 under the same experimental condition. Worth noting, although non-targeted uIONP/SN38 did not exhibited obvious cytotoxicity with the live cell population ranging from $72.5 \pm 2.2 \%$ to $87.1 \pm 1.3 \%$ (Fig. $\mathbf{5 a}$, $\mathbf{h}$ to $\mathbf{j}$ ), which was in accordance with the results in cell targeting experiment and cytotoxicity measurement, the dose-dependent decrease of live cell population suggested a minimal apoptotic effect on the cells under current condition, providing a reference for the dosing for future in vivo study.

\section{Discussion}

As one of the deadliest cancers, pancreatic cancer has been subject to limited regimen options with unsatisfying clinical outcome. The DNA topoisomerase inhibitor irinotecan, one of the major components in FOLFIRINOX, has attracted increasing attention in treating pancreatic cancer. Irinotecan has been shown to converted to its active metabolite SN38 through hydrolysis by carboxyl esterase[38], which induces apoptosis in cancer cells. However, the efficiency of irinotecan converting to SN38 is typically low $(<3 \%$ )[39]. With the attempts to directly deliver SN38 to solid tumors, various nano-formulations of SN38 have been reported in preclinical[40-42] and clinical studies[43]. Nanomaterials with smaller size has been shown to achieve better tumor penetration and accumulation than the larger counterparts in mouse model of pancreatic cancer[14]. Thus, the uIONPs with $3.5 \mathrm{~nm}$ as a drug carrier for SN38 hold great potential in improving the drug delivery efficiency comparing to the current SN38 nano-formulations. Moreover, the unique $\mathrm{T}_{1}-\mathrm{T}_{2}$ switchable MR contrast enhancement by uIONP based on its dispersion status[16, 44] allows for a more informative monitoring of the delivery of theranostic ulONPs to pancreatic tumors, with mono-dispersed uIONPs in tumor blood vessels showing bright $\mathrm{T}_{1}$-weighted contrast and aggregates of ulONPs delivered to the tumor exhibiting darkening $T_{2}$-weight enhancement. Furthermore, uIONPs have shown capable in renal clearance and faster degradations in organs of the reticuloendothelial system (RES) than larger IONPs[16], suggesting an improved biosafety profile in vivo. As the prolonged tissue retention of nanomaterials, particularly the metallic compartments, raised concerns for cytotoxicity[45], the uIONPs with faster body clearance may be more suitable for cancer therapy, allowing repeated dosing while causing less systemic toxicity and side effects, comparing to the larger counterparts.

One challenge in the drug delivery for pancreatic tumors is the dense stroma that prevents the penetration of nanomaterials to the tumor, making the tumor-cell-targeted ligand on the surface of nanomaterials less effective. As the IGF1R signaling pathway plays a critical role in cell proliferation and survival[46, 47], 
IGF1R is highly expressed by both pancreatic cancer cells and the stromal component[28, 29], making IGF-1 a suitable targeting ligand for pancreatic cancer. Moreover, the further elevated expression level of IGF1R on the tumor cells developing drug resistance[48, 49] makes the IGF1-uIONP a capable delivery system for delivering drug to those cells. However, IGF1R expression in human is ubiquitous[50], although not overexpressed in normal tissues[17]. Thus, the dosing of IGF1-uIONP/SN38 in future in vivo study needs to be optimized to ensure the delivery of sufficient drugs to the pancreatic tumors while minimizing the systemic cytotoxicity due to the over accumulation of IGF1-uIONP/SN38 in healthy organs resulted from IGF1R targeting.

\section{Conclusion}

In this study, we used a sub- $5 \mathrm{~nm}$ ulONP with amphiphilic polymer coating as the carrier to encapsulation highly potent yet hydrophobic DNA Topoisomerase I inhibitorSN38, with low molecular weight PEG facilitating the drug loading. The resultant uIONP/SN38 demonstrated highly stable drug loading and triggered release in tumor interstitial and lysosomal mimicking acidic environment. With IGF-1 conjugated as the ligand, the IGF1-uIONP drug delivery system for SN38 exhibited targeting specificity to the pancreatic cancer cells with overexpression of IGF1R, and improved inhibitory effect on pancreatic cancer cell growth over the free drug SN38, demonstrating its potential in treating pancreatic cancer.

\section{Declarations}

Ethics approval and consent to participate Not applicable.

Consent to publish Not applicable.

Availability of data and materials All data and materials in current study are available from the corresponding authors on reasonable request.

Competing Interests The authors have no relevant financial or non-financial interests to disclose.

Funding This work was funded by Guangdong Basic and Applied Basic Research Foundation [2020A1515010535], Shenzhen Fundamental Research Program [JCYJ20190808095413252], and Department of Innovation of Science and Technology of Longhua, Shenzhen.

Author Contributions Liya Wang and Yuancheng Li contributed to the study conception and design. Material preparation, data collection and analysis were performed by Peijia $\mathrm{Xu}$, Ting Xue, Jonathan Padelford, Alyssa Y Wu, and Xingkui Xue. The first draft of the manuscript was written by Peijia Xu and Ting Xue, and all authors commented on previous versions of the manuscript. All authors read and approved the final manuscript.

Acknowledgments Not applicable.

Compliance with Ethical Standards 
Disclosure of potential conflicts of interest All authors have no relevant financial or non-financial interests to disclose.

Research involving Human Participants and/or Animals Not applicable. This study does not involve any human participants or animals.

Informed consent Not applicable.

\section{References}

1. Kleeff, J., M. Korc, M. Apte, et al (2016) Pancreatic cancer. Nature Reviews Diease Primers 2:16022.

2. Park, W., A. Chawla, and E.M. O'Reilly (2021) Pancreatic cancer: a review. JAMA 326:851-862.

3. Burris 3rd, H.A., M.J. Moore, J. Andersen, et al (1997) Improvements in survival and clinical benefit with gemcitabine as first-line therapy for patients with advanced pancreas cancer: a randomized trial. Journal of Clinical Oncology 15:2403-2413.

4. Conroy, T., F. Desseigne, M. Ychou, et al (2011) FOLFIRINOX versus gemcitabine for metastatic pancreatic cancer. The New England Journal of Medicine 364:1817-1825.

5. Adiseshaiah, P.P., R.M. Crist, S.S. Hook, and S.E. McNeil (2016) Nanomedicine strategies to overcome the pathophysiological barriers of pancreatic cancer. Nature Reviews Clinical Oncology 13:750-765.

6. Huang, J., Y. Li, A. Orza, et al (2016) Magnetic nanoparticles facilitated drgu delivery for cancer therapy with targeted and image-guided approaches. Advanced Functional Materials 26:3818-3836.

7. Von Hoff, D.D., T. Ervin, F.P. Arena, et al (2013) Increased survival in pancreatic cancer with nabpaclitaxel plus gemcitabine. The New England Journal of Medicine 369:1691-1703.

8. Wang-Gillam, A., C.-P. LI, G. Bodoky, et al (2016) Nanoliposomal irinotecan with fluorouracil and folinic acid in metastatic pancreatic cancer after previous gemcitabine-based therapy (NAPOLI-1): a global, randomised, open-label, phase 3 trial. Lancet 387:545-557.

9. Kalra, A.V., J. Kim, S.G. Klinz, et al (2014) Preclinical activity of nanoliposomal irinotecan is governed by tumor deposition and intratumor prodrug conversion. Cancer Research 74:7003-7013.

10. Wang-Gillam, A., R.A. Hubner, J.T. Siveke, et al (2019) NAPOLI-1 phase 3 study of liposomal irinotecan in metastatic pancreatic cancer: Final overall survival analysis and characteristics of longterm survivors. European Journal of Cancer 108:78-87.

11. Cowan, R.W. and A. Maitra (2014) Genetic progression of pancreatic cancer. Cancer Journal 20:8084.

12. Rucki, A.A. and L. Zheng (2014) Pancreatic cancer stroma: understanding biology leads to new therapeutic strategies. World Journal of Gastroenterology 20:2237-2246.

13. Jain, R.K. and T. Stylianopoulos (2010) Delivering nanomedicine to solid tumors. Nature Reviews Clinical Oncology 7:653-664.

14. Cabral, H., Y. Matsumoto, K. Mizuno, et al (2011) Accumulation of sub-100 nm polymeric micelles in poorly permeable tumours depends on size. Nature Nanotechnology 6:815-823. 
15. Dadfar, S.M., K. Roemhild, N.I. Drude, S. von Stillfried, R. Knuchel, F. Kiessling, and T. Lammers (2019) Iron oxide nanoparticles: Diagnostic, therapeutic and theranostic applications. Advanced Drug Delivery Reviews 138:302-325.

16. Wang, L., J. Huang, H. Chen, et al (2017) Exerting enhanced permeability and retention effect driven delivery by ultrafine iron oxide nanoparticles with T1-T2 switchable magnetic resonance imaging contrast. ACS Nano 11:4582-4592.

17. Zhou, H., W. Qian, F.M. Uckun, et al (2015) IGF1 receptor targeted theranostic nanoparticles for targeted and image-guided therapy of pancreatic cancer. ACS Nano 9:7976-7991.

18. Lee, G.Y., W. Qian, L. Wang, et al (2013) Theranostic nanoparticles with controlled release of gemcitabine for targeted therapy and MRI of pancreatic cancer. ACS Nano 7:2078-2089.

19. Vangijzegem, T., D. Stanicki, and S. Laurent (2019) Magnetic iron oxide nanoparticles for drug delivery: applications and characteristics. Expert Opinion on Drug Delivery 16:69-78.

20. Shen, Z., A. Wu, and X. Chen (2017) Iron oxide nanoparticle based contrast agents for magnetic resonance imaging. Molecular Pharmaceutics 14:1352-1364.

21. Stephen, Z.R., F.M. Kievit, and M. Zhang (2011) Magnetite nanoparticles for medical MR imaging. Materials Today 14:330-338.

22. Ferguson, R.M., A.P. Khandhar, S.J. Kemp, et al (2015) Magnetic particle imaging with tailored iron oxide nanoparticle tracers. IEEE Transactions on Medical Imaging 34:1077-10894.

23. Talebloo, N., M. Gudi, N. Robertson, and P. Wang (2019) Magnetic particle imaging: current applications in biomedical research. Journal of Magnetic Resonance Imaging 51:1659-1668.

24. Xu, Y., H. Wu, J. Huang, et al (2020) Probing and enhancing ligand-mediated active targeting of tumors using sub-5 nm ultrafine iron oxide nanoparticles. Theranostics 10:2479-2494.

25. Xie, M., Y. Xu, J. Huang, Y. Li, L. Wang, L. Yang, and H. Mao (2020) Going even smaller: Engineering sub-5 nm nanoparticles for improved delivery, biocompatibility, and functionality. WIREs Nanomedicine and Nanobiotechnology 12:e1644.

26. de Man, F.M., A.K.L. Goey, R.H.N. van Schaik, R.H.J. Mathijssen, and S. Bins (2018) Individualization of irinotecan treatment: a review of pharmacokinetics, pharmacodynamics, and pharmacogenetics. Clinical Pharmacokinetics 57:1229-1254.

27. Ramesh, M., P. Ahlawat, and N.R. Srinivas (2010) Irinotecan and its active metabolite, SN-38: review of bioanalytical methods and recent update from clinical pharmacology perspectives. Biomedical Chromatography 24:104-123.

28. Valsecchi, M.E., M. McDonald, J.R. Brody, et al (2012) Epidermal growth factor receptor and insulinlike growth factor 1 receptor expression predict poor survival in pancreatic ductal adenocarcinoma. Cancer 118:3484-3493.

29. Ouban, A., P. Muraca, T. Yeatman, and D. Coppola (2003) Expression and distribution of insulin-like growth factor-1 receptor in human carcinomas. Human Pathology 34:803-808. 
30. Liu, Z., J.T. Robinson, X. Sun, and H. Dai (2008) PEGylated nanographene oxide for delivery of waterinsoluble cancer drugs. Journal of the American Chemical Society 130:10876-10877.

31. Huang, J., L. Wang, R. Lin, et al (2013) Casein-coated iron oxide nanoparticles for high MRI contrast enhancement and efficient cell targeting. ACS Applied Materials \& Interfaces 5:4632-4639.

32. Cooper, G.M., The Cell, 2nd edition. 2000, Sunderland (MA): Sinauer Associates.

33. Wagner, M. and H. Wiig (2015) Tumor interstitial fluid formation, characterization, and clinical implications. Frontiers in Oncology 5:115.

34. Li, Y., R. Lin, L. Wang, et al (2015) PEG-b-AGE polymer coated magnetic nanoparticle probes with facile functionalization and anti-fouling properites for reducing non-specific uptake and improving biomarker targeting. Journal of Materials Chemsitry B 3:3591-3603.

35. Zhou, Z., H. Chen, M. Lipowska, et al (2013) A dual-modal magnetic nanoparticle probe for preoperative and intraoperative mapping of sentinel lymph nodes by magnetic resonance and near infrared fluorescence imaging. Journal of Biomaterials Applications 28:100-111.

36. Yang, D., C. Liu, H. Piao, P. Quan, and L. Fang (2021) Enhanced drug loading in the drug-in-adhesive transdermal patch utilizing a drug-ionic liquid strategy: insight into the role of ionic hydrogen bonding. Molecular Pharmaceutics 18:1157-1166.

37. Rennick, J.J., A.P.R. Johnston, and R.G. Parton (2021) Key principles and methods for studying the endocytosis of biological and nanoparticle therapeutics. Nature Nanotechnology 16:266-276.

38. Smith, N.F., W.D. Figg, and A. Sparreboom (2006) Pharmacogenetics of irinotecan metabolism and transport: an update. Toxicology In Vitro 20:163-175.

39. Levesque, E., A.-S. Belanger, M. Harvey, et al (2013) Refining the UGT1A haplotype associated with irinotecan-induced hematological toxicity in metastatic colorectal cancer patients treated with 5fluorouracil/irinotecan-based regimens. The Journal of Pharmacology and Experimental Therapeutics 345:95-101.

40. Sepehri, N., H. Rouhani, F. Tavassolian, et al (2014) SN38 polymeric nanoparticles: in vitro cytotoxicity and in vivo antitumor efficacy in xenograft balb/c model with breast cancer versus irinotecan. International Journal of Pharmaceutics 471:485-497.

41. Nguyen, F., I. Alferiev, P. Guan, et al (2018) Enhanced intratumoral delivery of SN38 as a tocopherol oxyacetate prodrug using nanoparticles in a neuroblastoma xenograft model. Clinical Cancer Research 24:2585-2593.

42. Liu, X., Q. Huang, C. Yang, Q. Zhang, W. Chen, Y. Shen, and M. Sui (2017) A multi-stimuli responsive nanoparticulate SN38 prodrug for cancer chemotherapy. Journal of Materials Chemsitry B 5:661670.

43. Hamaguchi, T., A. Tsuji, K. Yamaguchi, et al (2018) A phase II study of NK012, a polymeric micelle formulation of SN-38, in unresectable, metastatic or recurrent colorectal cancer patients. Cancer Chemotherapy and Pharmacology 82:1021-1029.

44. Huang, J., L. Wang, X. Zhong, Y. Li, L. Yang, and H. Mao (2014) Facile non-hydrothermal synthesis of oligosaccharide coated sub- $5 \mathrm{~nm}$ magnetic iron oxide nanoparticles with dual MRI contrast 
enhancement effects. Journal of Materials Chemsitry B 2:5344-5351.

45. Longmire, M., P.L. Choyke, and H. Kobayashi (2008) Clearance properties of nano-sized particles and molecules as imaging agents: considerations and caveates. Nanomedicine (Lond) 3:703-717.

46. Hakuno, F. and S.-I. Takahashi (2018) IGF1 receptor signaling pathways. Journal of Molecular Endocrinology 61:T69-T86.

47. Iams, W. and C.M. Lovly (2015) Molecular pathways: clinical applications and future direction of insulin-like growth factor-1 receptor pathway blockade. Clinical Cancer Research 21:4270-4277.

48. Peled, N., M.W. Wynes, N. Ikeda, et al (2013) Insulin-like growth factor-1 receptor (IGF-1R) as a biomarker for resistance to the tyrosine kinase inhibitor gefitinib in non-small cell lung cancer. Cellular Oncology 36:277-288.

49. Suda, K., H. Mizuuchi, K. Sato, T. Takemoto, T. Iwasaki, and T. Mitsudomi (2014) The insulin-like growth factor 1 receptor causes acquired resistance to erlotinib in lung cancer cells with the wild-type epidermal growth factor receptor. International Journal of Cancer 135:1002-1006.

50. Delafontaine, P., Y.-H. Song, and Y. Li (2004) Expression, regulation, and function of IGF-1, IGF-1R, and IGF-1 binding proteins in blood vessels. Arteriosclerosis, Thrombosis, and Vascular Biology 24:435444.

\section{Figures}

\section{Figure 1}

(a) Hydrodynamic diameter of amphiphilic polymer coated ulONPs with a narrow distribution and the transmission electron microscopic image (inset). (b) UV-Vis spectroscopy of ulONPs (black trace), free SN38 (red trace) and uIONP/SN38 (blue trace), with the absorbance at $380 \mathrm{~nm}$ linearly correlated with the amount of ulONP/SN38 (inset). (c) Release of SN38 from uIONP/SN38 at the pH of 5.5, 6.5 and 7.4 within 24 hours.

\section{Figure 2}

(a) Change of SN38 loading efficiencies on uIONPs in respect to the PEG of different molecular weights used for drug encapsulation. The change of (b) hydrodynamic diameters and (c) zeta-potentials of ulONPs before and after encapsulation of SN38 with PEG550 and conjugation of IGF-1. 


\section{Figure 3}

Confocal fluorescence images of (a) to (c) MiaPaCa-2 human pancreatic cancer cells with overexpression of IGF1R and (d) to (f) HEK293 human embryonic kidney cells with normal expression of IGF1R after incubating with NIR830-labeled IGF1-uIONP/SN38. (g) to (i) confocal fluorescence images of MiaPaCa-2 cells after the incubation with non-targeted NIR830-uIONP/SN38.

\section{Figure 4}

AlamarBlue assay measured viability of (a) MiaPaCa-2 human pancreatic cancer cells and (b) HEK293 human embryonic kidney cells after treating with IGF1-uIONP/SN38, uIONP/SN38 and free SN38 at $37^{\circ} \mathrm{C}$ for 72 hours with the SN38 concentrations ranging from 0 to $2500 \mathrm{nM}$.

\section{Figure 5}

(a) Analysis of populations of live, apoptotic and dead cells after MiaPaCa-2 human pancreatic cancer cells were treated with IGF1-uIONP/SN38, uIONP/SN38 and free SN38. Representative flow cytometry analysis of gated cells stained by Annexin V FITC and 7-AAD showed the populations corresponding to viable and non-apoptotic (AnnexinV/77-AAD-), apoptotic (AnnexinV $\left.{ }^{+} / 7-A A D^{-}\right)$and dead (AnnexinV ${ }^{+} / 7-$ $\mathrm{AAD}^{+}$) cells after incubating MiaPaCa-2 cells with (b) to (d) IGF1-uIONP/SN38, (e) to (g) free SN38 and (h) to (j) ulONP/SN38.

\section{Supplementary Files}

This is a list of supplementary files associated with this preprint. Click to download.

- SupportingInformation.docx 